\title{
A Novel Approach to Segment Skin Lesions in Dermoscopic Images Based on a Deformable Model
}

\author{
Zhen Ma and João Manuel R. S. Tavares
}

\begin{abstract}
Dermoscopy is an imaging technique that has been widely used in the diagnosis of skin lesions. However, its accuracy largely depends on the dermatologist's experience; thus, computer-aided diagnosis techniques are required. In this paper, a novel approach based on a deformable model is proposed to handle the segmentation of skin lesions in dermoscopic images. The RGB color space is converted so that the color information contained in the images can be used effectively to differentiate normal skin and skin lesions; and the differences in the color channels are combined together to define the speed function and the stopping criterion of the deformable model. This novel approach is robust against the noise, and provides an effective and flexible segmentation. Two image databases were used to test the performance of the novel approach and the segmentation results obtained were satisfactory. Quantitative analysis on 250 dermoscopic images showed that the novel algorithm outperformed other state-of-the-art algorithms. Also, using comparative data, the reliability and the implementation issues of the approach are discussed in this work.
\end{abstract}

Index Terms-color spaces, image segmentation, level set method, medical imaging, melanoma.

\section{INTRODUCTION}

$\mathrm{M}$ ELANOMA refers to malignant tumors in melanocytes. Melanoma accounts for less than $20 \%$ among all cases of skin cancer, [1,2], but it is one of the three cancers with the highest mortality rate, and its incident is increasing rapidly in the Caucasian population [3-5]. However, if melanoma is detected in the early stages and treated properly, the survival rate is very high $[6,7]$.

Dermoscopy is a non-invasive imaging technique that has been developed to assist skin cancer diagnoses. A microscope with incident light and oil immersion is used to visualize the subsurface structures of the skin. This technique enables more details of colors and textures of the skin lesions to be

This work is funded by European Regional Development Funds (ERDF), through the Operational Programme 'Thematic Factors of Competitiveness' (COMPETE), and Portuguese Funds, through the Fundação para a Ciência e a Tecnologia (FCT), under the project: FCOMP-01-0124-FEDER-028160/PTDC/BBB- BMD/3088/2012. The first author also thanks FCT for the post-doc grant: SFRH/BPD/97844/2013.

Z. Ma and J. M. R. S. Tavares are members of the Instituto de Engenharia Mecânica e Gestão Industrial and the Faculdade de Engenharia da Universidade do Porto, Porto, Portugal (e-mail: zhen.ma@fe.up.pt; tavares@fe.up.pt). observed. Fig. 1 shows four examples of dermoscopic images. Dermoscopy improves the detection rate of melanomas considerably compared to inspection with the naked-eye whose accuracy is only $60 \%[6,8]$. Nevertheless, it has also been pointed out that the diagnostic accuracy using this technique largely depends on the dermatologist's experience $[9,10]$. In order to eliminate this subjectivity, computer-aided diagnoses (CAD) are needed. A common CAD system is composed of three steps: image segmentation, feature extraction, and classification. Given that the last two steps are based on the quantitative analysis of the segmented skin lesions, the accuracy of segmentation has a decisive influence on the whole CAD system.

The appearance of skin lesions in dermoscopic images may vary considerably depending on the skin condition. Also, the influence of hair, skin texture, and air bubbles may blur the boundary between the skin lesions and the surrounding healthy skin. Consequently, segmentation of dermoscopic images is a challenging task and a hurdle to overcome for an effective CAD system. Many algorithms have been proposed to solve this problem $[11,12]$. According to the technique used for segmentation, the current algorithms for dermoscopic images can be classified into three types: thresholding, clustering, and deformable models. The majority belongs to the first two types.

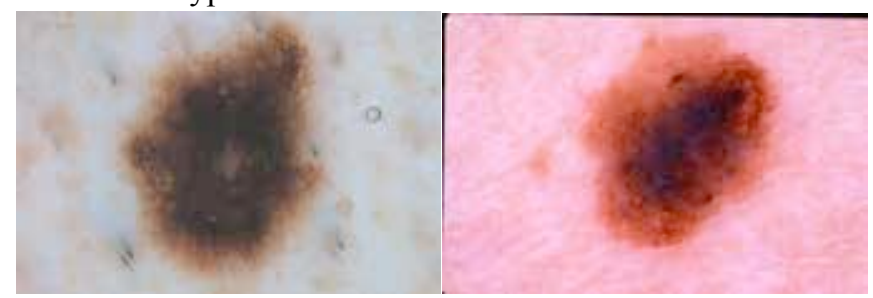

(a)

(b)

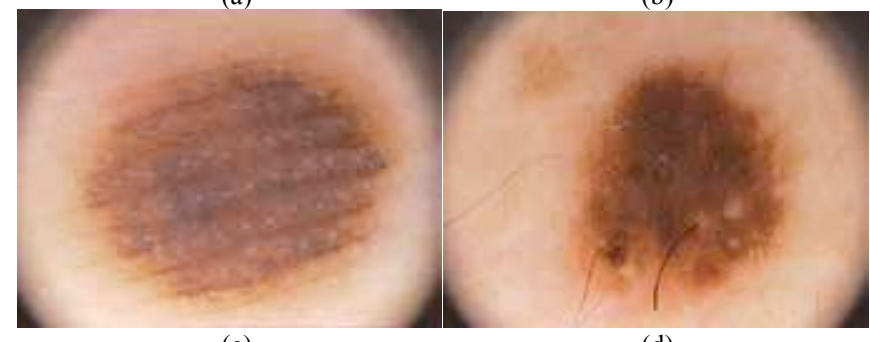

(c)

(d)

Fig. 1. (a) A dermoscopic image with a common nevi; (b)-(c) Two dermoscopic images with melanoma; (d) A dermoscopic image with an atypical nevi. 
The image segmentation algorithms based on thresholding depend on quantitative differences between the skin lesions and normal skin. For example, the fuzzy theory was combined with thresholding techniques for segmentation in [13], and an algorithm based on statistical region merging was proposed in [14]. A dermatologist-like tumor extraction algorithm (DTEA) was developed in [15] that combined the thresholding with the iterative region growing to carry out the segmentation; an improved version of this latter algorithm was presented in [16]. In [17], rough regions of skin lesions were first detected using a mixture model and local entropy techniques; then, a global thresholding technique based on Otsu's method was applied to refine the segmented regions. In [18], thresholding algorithms were combined and their segmentation results were fused together through an energy function to obtain a refined boundary of the skin lesions.

For algorithms based on clustering, pattern recognition techniques, such as clustering and supervised classification, were used to extract the features of skin lesions for segmentation. For example, a 2D color clustering algorithm was proposed in [19]; in that algorithm, a histogram was calculated from the two principal components of the CIE $L^{*} u * v^{*}$ color space, and a perceptron classifier was applied to obtain the centers of initial clusters; then a modified fuzzy C-means algorithm was used to segment the boundary of skin lesions. In [20], a neural network was trained based on the profiles of lesions in the training sets and the segmentation results were satisfactory with the assistance of a color normalization pre-processing step. An unsupervised algorithm based on a modified JSEG algorithm was proposed in [21]. In $[22,23]$ dynamic programming was used to solve the local minima and overlapping errors that appeared in the segmentation process. Statistical information, spatial interactions and color clustering techniques were incorporated to assist the segmentation in [24-27].

The algorithms proposed in [28-33] are examples of the third type. By using a deformable model, the segmentation is treated as a curve evolution and the final status of the moving contour(s) is defined as the object boundary. Compared to the first two types, the segmentation algorithms based on deformable models are more flexible to handle the influence of noise, artifacts, and variations in illumination and color, and their performance is more robust when segmenting images acquired under complex imaging conditions. Comparisons and reviews of different segmentation algorithms can be found in [12, 34-37].

In this study, we focus on the segmentation of skin lesions in dermoscopic images and propose a novel approach based on a deformable model. Following the statistical features of dermoscopic images in different color spaces, the contrasts between the lightness and saturation of the skin lesions and the surrounding normal healthy skin were used as the segmentation clues and were combined to generate the region-based external forces. The color information of the dermoscopic images was used to assist the segmentation and prevent over-contraction of the curve evolution. Therefore, the initial curve can move towards the boundary of skin lesions in a robust way.

In the next section, color spaces and deformable models are reviewed; then, in Section 3, the proposed approach is introduced, including the definitions of the initial conditions, speed function and stopping strategy; afterwards, numerical tests are presented, and based on the quantitative analysis, implementation issues of the algorithm are discussed. Finally, in the last section, the conclusions and perspectives of future work are indicated.

\section{BACKGROUND}

Dermoscopic images are normally acquired by a hand-held dermascope, with the color information represented in the 24-bit RGB color space for display purposes. However, the majority of the current segmentation algorithms were developed for gray-scale images; a straight-forward method to segment the skin lesions is to discard the color information and convert the color dermoscopic images to gray-scale images, in such a way the image intensity of each pixel is calculated through a weighted combination of the three RGB channels. Nevertheless, the appearance of skin lesions may vary considerably depending on the skin condition, and in some cases its main distinction to the normal skin is the chromaticity that will be either lost or weakened when the color information is treated as a single intensity value. Therefore, the RGB color space should be converted to suitable color spaces so that the color information can be used more effectively for segmentation.

\section{A. Color Spaces}

The RGB color space is the most common color space used to store color information. Despite its popularity for display purposes, the RGB color representation is unsuitable for many image segmentation problems; one of the main reasons for this difficulty is that the three color channels contain correlated information, and when the color varies, the change of each component is not linear and difficult to predict. Therefore, it is hard to measure the difference between two colors in the RGB color space. 
In order to solve this problem and to use the color information more effectively, two color spaces of the CIE system - $L^{*} a^{*} b^{*}$ and $L^{*} u^{*} v^{*}$ models have been adopted. Both of them are variations of the CIE XYZ color space and are referred to as uniform color models. In these two color spaces, the lightness component is separated from the color expression into channel $L$ with values ranging from 0 to 100; the separation is a big advantage when processing dermoscopic images as the perceptual difference between colors is often influenced by lightness variations. The values of $u^{*}$ and $v^{*}$ in the $L^{*} u^{*} v^{*}$ model are the chrominance coordinates $\left(u^{*}, v^{*}\right)$ representing the position of the color in the uniform chromaticity scale (UCS) diagram. The value of $a^{*}$ in the $L^{*} a^{*} b^{*}$ model stands for the position of the color between magenta (positive direction) and green (negative direction); and the value of $b^{*}$ stands for the position of the color between yellow (positive direction) and blue (negative direction). Formulas that are used to calculate these channels can be found in $[38,39]$.

Fig. 2 illustrates the different channels in the converted color spaces by mapping the channel values into the range $[0,255]$; in these channels the differences between the normal skin and skin lesions can be seen clearly.

\section{B. Geometric Deformable Models}

Deformable models are effective techniques for image segmentation and have a wide variety of applications such as in medical image processing and analysis [11]. The main idea of this technique is to model the segmentation as a process of a curve evolution; thus, a proper speed function needs to be defined with which the initial curve can be driven to the desired boundary. Based on the tracking approach of curve evolution, deformable models can be divided into parametric models and geometric models. The level set method [40] was adopted in the geometric models to facilitate the computations: instead of tracking the movement of each curve point, the curve is embedded into a higher-dimensional level set function as its zero level set. This implicit representation decreases the computational complexity for tracking considerably. For two-dimensional image processing, the equation of motion for a geometric deformable model is normally a level set equation written as:

$\frac{\partial \phi}{\partial t}+F|\nabla \phi|=0$

where $\phi(x, y, t)$ is the level set function with $(x, y)$ the coordinates and $\phi(x, y, t)=0$ representing the curve at the time $t ; F$ is the speed function with which the moving curve can stop or reach a stable status at the boundary of the focused object. The level set function $\phi(x, y, 0)$ is usually defined as the signed distance function to the initial curves.

Compared with other image segmentation techniques, deformable models have several advantages when applied to dermoscopic images: the implicit representation of the moving curve is a way to obtain the regions and the boundaries of the skin lesions simultaneously, which is a desirable feature for shape and color analysis; the robustness against the influence of noise with the smoothing effects of the internal forces

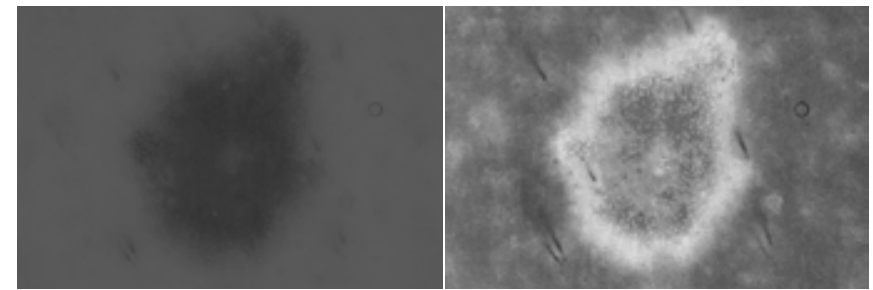

(a) (b)

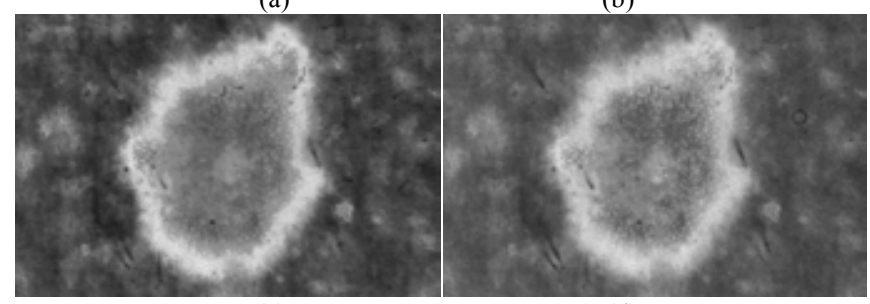

(c)

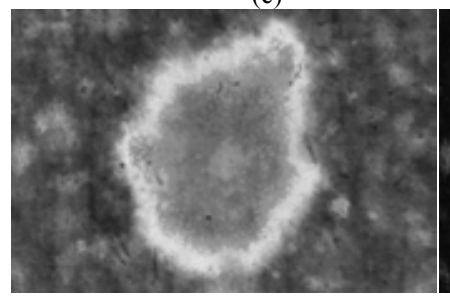

(e)

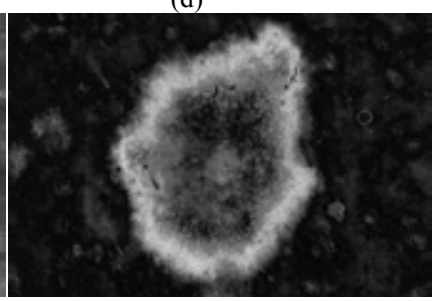

(f)

Fig. 2. (a) The lightness channel in the $C I E$ color systems of the image in Fig. 1a; (b)-(c) The $a$ and $b$ channels of the image in Fig. 1a with their values mapped to the range [0,255] for illustration purpose; (d)-(e) The $u$ and $v$ channels of the image in Fig la mapped to the range $[0,255]$. (f) The saturation channel of the image in Fig. 1a mapped to the range $[0,255]$.

during the curve evolution; and strategies can be integrated to guarantee that the boundaries obtained have the desired properties, for example, to have the same topology as the initial curve [41].

\section{SEGMENTATION}

Deformable models are normally semi-automatic. However, there are two issues that can appreciably affect their performance and therefore, need to be well defined: the initial conditions and the values of the parameters used. The following strategies were adopted in the proposed approach to achieve an effective segmentation of skin lesions in dermoscopic images.

\section{A. Initial Curve}

There are no general requirements on the position of the initial curves in a geometric deformable model, since the evolution and moving direction are determined by the speed function. However, for the segmentation of skin lesions, given that the colors of skin lesions are frequently inhomogeneous, the evolving curves can easily be attracted to the wrong inner boundaries if they move inside the skin lesion regions. On the other hand, normal healthy skin has a comparably homogeneous color distribution. Hence, the curve evolution is limited to contraction in the novel approach. In this way, the position of the initial curve is determined by the location of the skin lesion and is required to cover the entire region of the skin lesion. The initial curve will then move inwards until it arrives at the boundary of the skin lesion where the lightness and color are different than normal healthy skin. 
Due to the complex imaging background of dermoscopic images, the initial curve is defined manually. The values of the level set function $\phi(x, y, 0)$ are then defined as:

$\phi(x, y, 0)=\left\{\begin{array}{lr}d(x, y) & \text { if the pixel is inside } C \\ -d(x, y) & \text { otherwise }\end{array}\right.$,

where $d(x, y)$ is the Euclidean distance of the pixel $(x, y)$ to the contour $C$; and the internal and external regions of the evolving curve at the time $t$ can be written as:

$\Omega_{I}=\{(x, y) \mid \phi(x, y, t)>0\}$,

$\Omega_{E}=\{(x, y) \mid \phi(x, y, t) \leq 0\}$.

\section{B. Speed Function}

The CIE $L^{*} a^{*} b^{*}$ and $L^{*} u^{*} v^{*}$ color models provide approximately uniform perceptual descriptions of colors. Among the five channels in the two spaces, the lightness channel provides an important clue for segmentation. However, exclusively using this channel can cause unreliable segmentation, a similar problem occurs when using a gray-scale image. Therefore the color information is necessary for a correct segmentation. The $a^{*}, b^{*}, u^{*}$ and $v^{*}$ channels are colour coordinates, and are unable to express in a simple way the chromatic differences between normal skin and skin lesions quantitatively; also, various parameters are needed to map the chromatic changes when using these channels. These factors can lead to less reliable segmentations and they are more sensitive to imaging conditions; hence, these four channels are inappropriate to define the speed function for segment dermoscopic images. Consequently, a measure is needed that can combine the information from the lightness and the colorfulness contained in the dermoscopic image. Thus color saturation is adopted to fulfill this bridging role.

Saturation is a measure that describes the colorfulness of a color relative to its lightness. This channel appears in many color space models, such as HSL, HSV, and HSI; nonetheless, it is not officially defined in the $C I E$ system. There are various definitions and formulas to calculate this channel; a widely used one in computer vision is:

$S=\left\{\begin{array}{cr}0 & \text { if } R+G+B=0 \\ 1-\frac{\min (R, G, B)}{(R+G+B) / 3} & \text { otherwise }\end{array}\right.$.

Let's make a general assumption that the lightness and saturation of the normal skin follow Gaussian distributions; then, with the above definitions, the speed function of the deformable model is proposed as:

$F(x, y)=P_{L}(x, y) * P_{S}(x, y) *(1+\kappa)$,

where $\kappa=\nabla\left(\frac{\nabla \phi}{|\nabla \phi|}\right)$ is the curve curvature that serves as the internal force to regularize the geometric properties of the moving curve, and

$P_{L}(x, y)=\frac{1}{\sqrt{2 \pi} \sigma_{L}} \exp \left(-\frac{\left(L(x, y)-\mu_{L}\right)^{2}}{2 \sigma_{L}^{2}}\right)$,

$P_{S}(x, y)=\frac{1}{\sqrt{2 \pi} \sigma_{S}} \exp \left(-\frac{\left(S(x, y)-\mu_{S}\right)^{2}}{2 \sigma_{S}^{2}}\right)$,

where $\left(\mu_{L}, \sigma_{L}\right)$ and $\left(\mu_{S}, \sigma_{S}\right)$ are the means and standard deviations of the lightness and saturation values of the normal skin respectively.

With the initial curve covering the skin lesion, such a speed function will lead the evolving curve to contract to the places where either the lightness or the saturation is appreciably different from normal skin. The definition of the speed function in (6) includes the statistical information of lightness and saturation channels of normal skin. Nevertheless, these values are unknown prior to the segmentation. To obtain an approximation of these four values, the Otsu's method [42] is applied to binarize the image based on the lightness channel. Otsu's method is adopted here because in most cases, it can provide a suitable preliminary classification based on the lightness difference between normal skin and skin lesions [16, $21,36]$. In order to facilitate this process the intensity function $I$ in the binary image is defined as:

$I(x, y)=\left\{\begin{array}{c}0 \text { if pixel }(x, y) \text { represents normal skin } \\ 255 \text { if pixel }(x, y) \text { represents skin lesion }\end{array}\right.$

Correspondingly, the region of normal skin $\Omega_{0}$ and the region of skin lesions $\Omega_{1}$ can be written as:

$\Omega_{0}=\{(x, y) \mid I(x, y)=0\}$,

$\Omega_{1}=\{(x, y) \mid I(x, y)=255\}$,

The values of $\mu_{L}, \sigma_{L}, \mu_{S}, \sigma_{S}$ are then calculated in a narrow band next to the initial curve defined as follows:

$\Omega_{0}^{\prime}=\{(x, y) \mid-50<\phi(x, y, 0)<0\} \cap \Omega_{0}$,

where $\phi(x, y, 0)$ is the initial level set function defined in (2). The region $\Omega_{0}^{\prime}$ is composed of pixels that are classified as representing normal skin in the binary image of (9) and is located next to the initial curve; given that the skin lesions are completely inside the initial curve, this region can provide an approximation of the statistical distributions of the lightness and saturation of the normal skin. These statistical values are then updated along with the curve evolution.

\section{Evolution}

The speed function in (6) involves only the information of normal skin, as the appearance of the skin lesions are very varied. As reviewed in Section II, the coordinates $\left(a^{*}, b^{*}\right)$ and $\left(u^{*}, v^{*}\right)$ in the $C I E L^{*} a^{*} b^{*}$ and $L^{*} u^{*} v^{*}$ color spaces describe the positions of a color relative to the color base and diagram. The locations of these coordinates reflect the major perceptual difference between the normal skin and skin lesions; hence, the coordinates of pixels representing normal skin should be near to each other and have considerable distance to the ones representing skin lesions. Accordingly, the image pixels can be classified into two groups based on their distances to the centroids of normal skin and skin lesions in the two color coordinate systems.

However, a similar problem as the one in Section 3.2 arises: the centroids of the two groups are unknown. Also, the binary image from the Otsu's method is unsuitable to be used here to calculate the centroids, because the centers obtained with such a preliminary classification may have large deviations to the true ones and cause incorrect segmentations. Instead, since the skin lesions are inside the curves, a neighboring external region $\Omega_{0}^{(t)}$ of the evolving curve at the time $t$ is used to calculate the centroid of normal skin in the color space, and the internal region $\Omega_{1}^{(t)}$ of the curve is used to calculate the centroid of the skin lesions:

$\Omega_{0}^{(t)}=\{(x, y) \mid-50<\phi(x, y, t)<0\}$, 
$\Omega_{1}^{(t)}=\{(x, y) \mid \phi(x, y, t)>0\}$,

Accordingly, the centroids $\left(a_{0}^{*}, b_{0}^{*}\right)$ and $\left(u_{0}^{*}, v_{0}^{*}\right)$ of the normal skin, and $\left(a_{1}^{*}, b_{1}^{*}\right)$ and $\left(u_{1}^{*}, v_{1}^{*}\right)$ of the skin lesions are calculated as:

$a_{0}^{*}=\frac{1}{\left\|\Omega_{0}^{(t)}\right\|} \sum_{p \in \Omega_{0}^{(t)}} a_{p}^{*}, b_{0}^{*}=\frac{1}{\left\|\Omega_{0}^{(t)}\right\|} \sum_{p \in \Omega_{0}^{(t)}} b_{p}^{*}$,

$u_{0}^{*}=\frac{1}{\left\|\Omega_{0}^{(t)}\right\|} \sum_{p \in \Omega_{0}^{(t)}} u_{p}^{*}, v_{0}^{*}=\frac{1}{\left\|\Omega_{0}^{(t)}\right\|} \sum_{p \in \Omega_{0}^{(t)}} v_{p}^{*}$,

$a_{1}^{*}=\frac{1}{\left\|\Omega_{1}^{(t)}\right\|} \sum_{p \in \Omega_{1}^{(t)}} a_{p}^{*}, b_{1}^{*}=\frac{1}{\left\|\Omega_{1}^{(t)}\right\|} \sum_{p \in \Omega_{1}^{(t)}} b_{p}^{*}$,

$u_{1}^{*}=\frac{1}{\left\|\Omega_{1}^{(t)}\right\|} \sum_{p \in \Omega_{1}^{(t)}} u_{p}^{*}, v_{1}^{*}=\frac{1}{\left\|\Omega_{1}^{(t)}\right\|} \sum_{p \in \Omega_{1}^{(t)}} v_{p}^{*}$.

Along with the contraction of the curve, the centroids of the skin lesions and the surrounding normal skin will become more accurate. Meanwhile, a binary image can be generated using the following rule:

$I^{(t)}(x, y)=$

$\left\{\begin{array}{cr}0 & \text { if } d_{0}\left(a^{*}, b^{*}\right)<d_{1}\left(a^{*}, b^{*}\right) \text { and } d_{0}{ }^{\prime}\left(u^{*}, v^{*}\right)<d_{1}{ }^{\prime}\left(u^{*}, v^{*}\right) \\ 255 & \text { otherwise }\end{array}\right.$

where $a^{*}, b^{*}, u^{*}, v^{*}$ are the values of the corresponding channels of the pixel $(x, y)$, and $d_{0}, d_{1}, d_{0}{ }^{\prime}, d_{1}{ }^{\prime}$ are the Euclidean distances to the centroids of the normal skin and the skin lesions in the CIE $L^{*} a^{*} b^{*}$ and $C I E L^{*} u^{*} v^{*}$ color spaces, respectively, which are calculated as:

$d_{0}\left(a^{*}, b^{*}\right)=\sqrt{\left(a^{*}-a_{0}^{*}\right)^{2}+\left(b^{*}-b_{0}^{*}\right)^{2}}$,

$d_{1}\left(a^{*}, b^{*}\right)=\sqrt{\left(a^{*}-a_{1}^{*}\right)^{2}+\left(b^{*}-b_{1}^{*}\right)^{2}}$,

$d_{0}{ }^{\prime}\left(u^{*}, v^{*}\right)=\sqrt{\left(u^{*}-u_{0}^{*}\right)^{2}+\left(v^{*}-v_{0}^{*}\right)^{2}}$,

$d_{1}{ }^{\prime}\left(u^{*}, v^{*}\right)=\sqrt{\left(u^{*}-u_{1}^{*}\right)^{2}+\left(v^{*}-v_{1}^{*}\right)^{2}}$.

Using these definitions, only the pixels of the colors that are similar to normal skin in both the $a^{*}-b^{*}$ and $u^{*}-v^{*}$ planes are assigned to the black color.

The binary image $I^{(t)}$ actually provides a classification of the image pixels according to their colors; based on this classification, the distribution of the saturation values of normal skin around the skin lesions can be more suitable than that from the region defined in (12). Hence, the mean and standard deviation of normal skin $\left(\mu_{S}, \sigma_{S}\right)$ are calculated and updated during the evolution in a sub-region of $\Omega_{0}^{(t)}$ defined as:

$\Omega_{0}^{\prime(t)}=\left\{(x, y) \mid I^{(t)}(x, y)=0\right\} \cap \Omega_{0}^{(t)}$.

Then, the speed values defined in (6) are updated in order to guide the curve towards the segmentation more accurately.

\section{Stopping Criterion}

In the ideal situation, the curve will move inwards with the speed function defined in (6) until it arrives at the position where either the lightness or the saturation is different from normal skin. The quick decrease of the speed value will slow down the movement of the curve and let it achieve a stable status. However, due to the considerable variations in the lightness and saturation values of normal skin, when the curve arrives at a skin lesion, the decrease of speed values may not be enough to attract the curve to the boundary; and further evolution will let the curve leak into the skin lesions. Therefore, strategies are needed to avoid this.

The white regions in the binary image $I^{(t)}$ represent the skin lesions, and along with the curve evolution, this classification will be refined and will concentrate on the real boundaries of the skin lesions. To assure that the curve moves slowly when close to the boundary of a skin lesion, the speed function in (6) is modified to:

$F^{*}(x, y)=\left\{\begin{array}{c}\alpha F(x, y) \text { if } I^{(t)}(x, y)=255 \\ F(x, y) \text { if } I^{(t)}(x, y)=0\end{array}\right.$,

where $\alpha \in[0,1]$ is a penalty to the speed values of the pixels that are classified as the skin lesions at the time $t$. The modified speed function can attach the moving curve to the possible boundary. The parameter $\alpha$ can be viewed as the color sensitivity of the segmentation: when $\alpha$ approaches zero, the curve movement will be affected more by the changes of color and will be more sensitive to the influence of noise and the initial classification $I^{(0)}$; with the increase of $\alpha$, the penalty to the speed function is weakened, and the curve will have a greater possibility to pass the wrongly classified pixels and cause leakage. From another point of view, this parameter is a mimic of the perceptual differences among individuals, and by adjusting its value the segmentation becomes more flexible to follow the dermatologists' evaluation.

Additionally, once a curve moves into the region of a skin lesion, the different colors of the skin lesion will dramatically change the centroids of normal skin and the skin lesions in the two color spaces. As a result, there will be a considerable amount of pixels misclassified as the skin lesions, and the binary image $I^{(t)}$ has a tendency to invert the black and white regions. Based on this clue, the evolution is stopped once the area of the skin lesions in $I^{(t)}$ increases appreciably.

Besides the above strategy, an index $L_{-} D^{(t)}$ is defined as:

$L_{-} D^{(t)}=\left|\overline{L_{\Omega_{0}^{(t)}}}-\overline{L_{\Omega_{1}^{(t)}}}\right|$,

where $\overline{L_{\Omega_{0}^{(t)}}}$ and $\overline{L_{\Omega_{1}^{(t)}}}$ are the mean lightness values of the regions $\Omega_{0}^{(t)}$ and $\Omega_{1}^{(t)}$ defined in (13) and (14), respectively. This index reflects the difference of the mean lightness values of the internal and external regions of the moving curve, and its value should increase along with the curve evolution. Hence, once the curve moves into the region of skin lesions, its value has a tendency to decrease; then, the curve evolution is stopped.

\section{E. Summary of Procedure}


In the proposed approach, the color information is incorporated into the segmentation process and is used to assist the determination of the final status of evolution. Fig. 3 shows the process of segmentation using the image shown in Fig. 1a. The procedure of the approach can be outlined as follows:

Preprocessing:

Smooth the original image by applying a median filter to each channel of the RGB space.

\section{Initialization:}

Set $t=0$,

Define the initial curve;

Compute the initial level set function $\phi(x, y, 0)$ using (2);

Apply the Otsu's method on the $L^{*}$ channel in the CIE $L * a * b *$ space;

Compute the statistical values $\mu_{L}, \sigma_{L}, \mu_{S}, \sigma_{S}$ based on the region $\Omega_{0}^{\prime}$ defined in (12);

\section{Repeat:}

Set $t=t+1$,

Compute $\phi(x, y, t)$ according to (1);

Calculate the spatial color centers $\left(a_{0}^{*}, b_{0}^{*}\right),\left(u_{0}^{*}, v_{0}^{*}\right),\left(a_{1}^{*}, b_{1}^{*}\right)$, and $\left(u_{1}^{*}, v_{1}^{*}\right)$ using (15) - (18);

Build the binary image $I^{(t)}$ with (19) and update the values $\mu_{S}, \sigma_{S}$ with the region $\Omega^{\prime(t)}$ defined in (24);

Compute the speed function $F$ with (25).

\section{Until:}

$t>t_{\text {max }}$, or

The curve has achieved a stable status, or

The area of the white region in $I^{(t)}$ has a tendency to increase, and $L_{-} D^{(t)}$ in (26) has a tendency to decrease.

\section{EXPERIMENT\& DISCUSSION}

Due to inter-observer errors, the ground truth of lesion boundaries in dermoscopic images does not exist in normal practice. Therefore, the comparison with the manual segmentation by experienced technicians is a common way to evaluate the performance of an algorithm and was adopted in this study.

\section{A. Numerical Tests}

Two image databases were used to test the effectiveness of the proposed algorithm: one is the database used in [18], which is composed of 90 dermoscopic images with 23 images diagnosed as melanomas and 67 as benign nevi; the other is a challenging dermoscopic image database called PH2 [43], in which 160 images captured the complete region of skin lesions with 8 images diagnosed as melanoma and 152 as either typical nevi or atypical nevi. Hence, a total of 250 dermoscopic images were used for numerical tests. In order to perform the quantitative analysis, let's suppose that $C$ is the boundary of the skin lesions; then a binary image $I_{C}$ can be defined correspondingly as:

$I_{C}(x, y)=\left\{\begin{aligned} 0, & \text { if }(x, y) \text { is outside } C \\ 255, & \text { if }(x, y) \text { is inside } C\end{aligned}\right.$

Afterwards, the region of the skin lesions is:

$\Omega=\left\{(x, y) \mid I_{C}(x, y)=255\right\}$

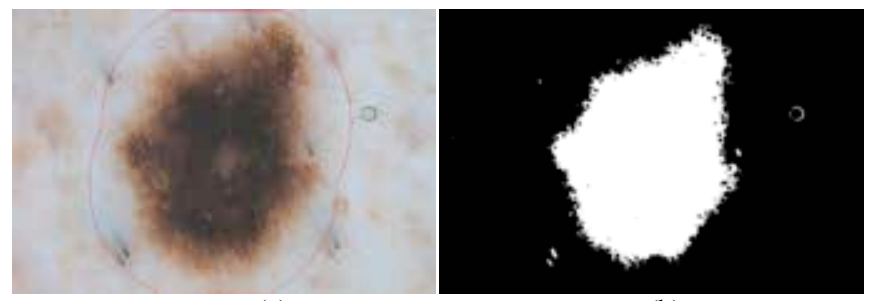

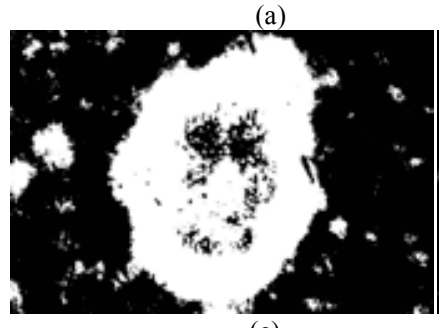

(c)

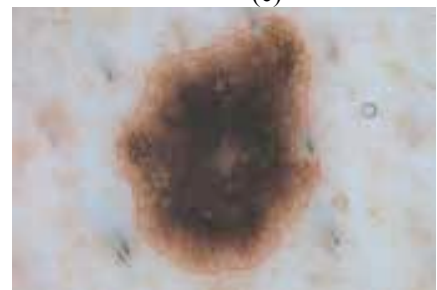

(e)

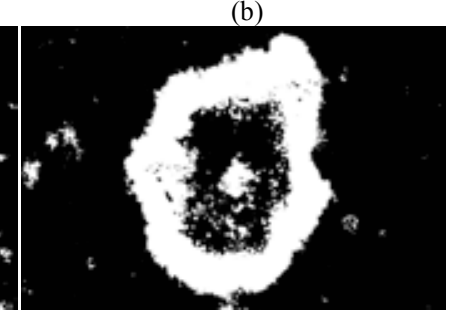

(d)

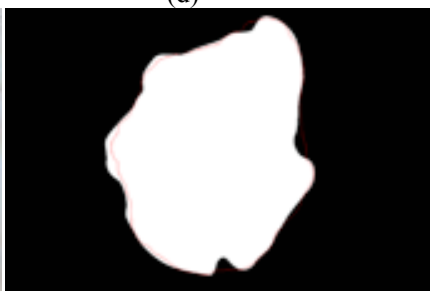

(f)
Fig. 3. An illustration of the proposed approach with the image in Fig. 1a: (a) the initial curve (red contour); (b) the binary image generated with (18), which is obtained by applying the Otsu's method; (c) the binary image generated with (28) based on the color coordinates when the curve begins to evolve in Fig. 3a; (d) the binary image generated with (28) when the curve achieves stable status in Fig. 3e; (e) the final location of the curve; (f) The boundary obtained overlapped with the ground truth of the skin lesion (white area), and the comparison values were $D\left(I_{0}, I_{1}\right)=0.0507, D_{1}\left(C_{0}, C_{1}\right)=$ $6.7629, D_{1}\left(C_{0}, C_{1}\right)=6.268$.

and the area of skin lesions can be defined as:

$\operatorname{Area}(I)=\|\Omega\|$.

With these definitions, the following three measures were used to evaluate the difference between the ground truth and the result obtained:

$D\left(I_{0}, I_{1}\right)=\operatorname{Area}\left(I_{0} \oplus I_{1}\right) / \operatorname{Area}\left(I_{0}\right)$,

$D_{1}\left(C_{0}, C_{1}\right)=\overline{D\left(p, C_{1}\right)}, p \in C_{0}$,

$D_{2}\left(C_{0}, C_{1}\right)=\overline{D\left(p, C_{0}\right)}, p \in C_{1}$,

where $C_{0}$ is the ground true of the boundary, $C_{1}$ is the boundary obtained by the algorithm, $I_{0}$ and $I_{1}$ are the binary images defined by $C_{0}$ and $C_{1}$ using (27), $\oplus$ is the exclusive or (XOR) operator, $D(p, C)$ is the point-to-contour distance that can be calculated as $D(p, C)=\min _{p^{*} \in C} d\left(p, p^{*}\right)$ with $d\left(p, p^{*}\right)$ the Euclidean distance between the two points, which is a similar way used in the Hausdorff distance to define such a point-to-contour distance, and $\overline{D(p, C)}$ is the mean of this value. The value of $D\left(I_{0}, I_{1}\right)$ reflects the deviation of the segmented regions of the skin lesion; while the contour-to-contour distance in (31) and (32) reflect the differences between the boundaries.

The comparison of the results with $\alpha=0.5$ using the measures in (30) - (32) are listed in Table I. Although the performance of the proposed algorithm using the second image database is not as good as the performance with the database in [18], it still achieves a very satisfactory result 
TABLE I

QUANTITATIVE ANALYSIS OF THE PROPOSED APPROACH

\begin{tabular}{|c|c|c|c|c|c|c|}
\hline \multirow{2}{*}{ Type } & \multicolumn{2}{|c|}{$D\left(I_{0}, I_{1}\right)$} & \multicolumn{2}{|c|}{$D_{1}\left(C_{0}, C_{1}\right)$} & \multicolumn{2}{|c|}{$D_{2}\left(C_{0}, C_{1}\right)$} \\
\hline & $\mu(\%)$ & $\sigma(\%)$ & $\mu$ & $\sigma$ & $\mu$ & $\sigma$ \\
\hline Benign $^{1}$ & 10.03 & 4.34 & 8.91 & 4.59 & 8.59 & 4.20 \\
\hline Melanoma $^{1}$ & 13.11 & 4.88 & 11.44 & 5.82 & 10.14 & 5.42 \\
\hline All $^{1}$ & 10.82 & 4.66 & 9.55 & 5.03 & 8.99 & 4.56 \\
\hline Benign $^{2}$ & 13.91 & 7.79 & 10.35 & 6.03 & 9.67 & 5.73 \\
\hline Melanoma $^{2}$ & 14.16 & 8.07 & 11.43 & 5.52 & 11.67 & 7.49 \\
\hline $\mathrm{All}^{2}$ & 13.92 & 7.78 & 10.41 & 5.99 & 9.77 & 5.82 \\
\hline
\end{tabular}

${ }^{1}$ Images from the database in [18].

${ }^{2}$ Images from the $\mathrm{PH} 2$ database.

TABLE II

COMPARISON DATA OF THE NINE STATE-OF-THE-ART ALGORITHMS IN [18]

\begin{tabular}{|c|c|c|c|c|c|c|}
\hline \multirow{2}{*}{ Algorithm } & \multicolumn{2}{|c|}{ Benign } & \multicolumn{2}{|c|}{ Melanoma } & \multicolumn{2}{|c|}{ All } \\
\hline & $\mu(\%)$ & $\sigma(\%)$ & $\mu(\%)$ & $\sigma(\%)$ & $\mu(\%)$ & $\sigma(\%)$ \\
\hline [14] & 11.38 & 6.23 & 10.29 & 5.84 & 11.11 & 6.12 \\
\hline [16] & 10.51 & 4.73 & 11.85 & 6.00 & 10.86 & 5.08 \\
\hline [19] & 22.99 & 12.61 & 28.31 & 15.25 & 24.35 & 13.45 \\
\hline [21] & 10.83 & 6.36 & 13.75 & 7.59 & 11.58 & 6.77 \\
\hline [28] & 13.69 & 5.59 & 19.34 & 9.33 & 15.13 & 7.13 \\
\hline [35] & 11.53 & 9.74 & 13.29 & 7.42 & 11.98 & 9.19 \\
\hline [20] & 10.07 & 4.34 & 18.17 & 26.96 & 12.14 & 14.36 \\
\hline [44] & 12.95 & 6.17 & 16.93 & 7.16 & 13.96 & 6.63 \\
\hline [45] & 21.56 & 25.19 & 23.51 & 16.06 & 22.06 & 23.13 \\
\hline New one & 10.03 & 4.34 & 13.11 & 4.88 & 10.82 & 4.66 \\
\hline
\end{tabular}

$\mu$ and $\sigma$ are the mean and standard deviation of the measure $D\left(I_{0}, I_{1}\right)$ defined in (30).

given that the images in $\mathrm{PH} 2$ have more complex backgrounds and contain more complicated skin conditions. To make the evaluation more comprehensive and unbiased, Table II lists the data presented in [18] that describes the performance of nine state-of-the-art algorithms $[14,16,19,21,28,35,20,44$, $45]$ on the first database with the measure defined in (30). The nine algorithms cover all the three major effective segmentation techniques developed for dermoscopic images. Among them, the ones in [14] and [44] are thresholding-based; [16], [19], [20], [21], [35] and [45] are algorithms based on clustering techniques; and in [28] the algorithm is based on a deformable model. This table shows that the proposed approach has the smallest mean error percentage on images with benign skin lesions, and it achieved the best overall performance in the first database.

Fig. 4 illustrates several examples in the two image databases, from which one can see the robustness of the proposed approach against the different imaging conditions and distinct types of skin lesions.

\section{B. Parameters and Initial Conditions}

Tests on different image databases can show the adaptability of the proposed algorithm to distinct imaging conditions and illustrate its robustness against their variances. To show the consistency of the approach, the value of $\alpha$ in (25) was fixed as 0.5 for both image databases. A notable point is that for a specific image, changing this value can enhance the similarity of the boundary obtained to the manual segmentation which was used as the ground truth. A case in point is shown in Fig. 5c where the boundary obtained is very similar to the manual segmentation with a smaller $\alpha$ and the

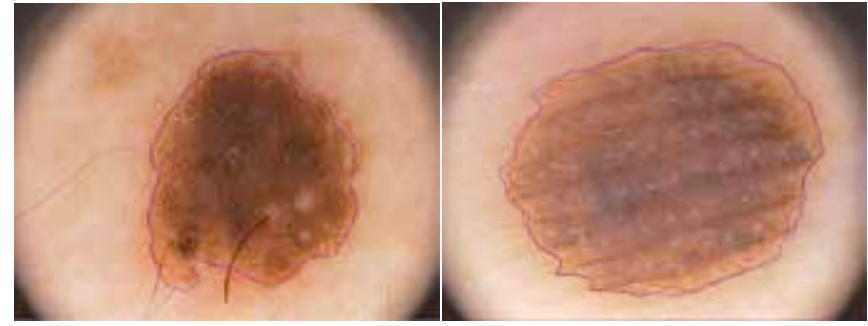

(a)

(b)

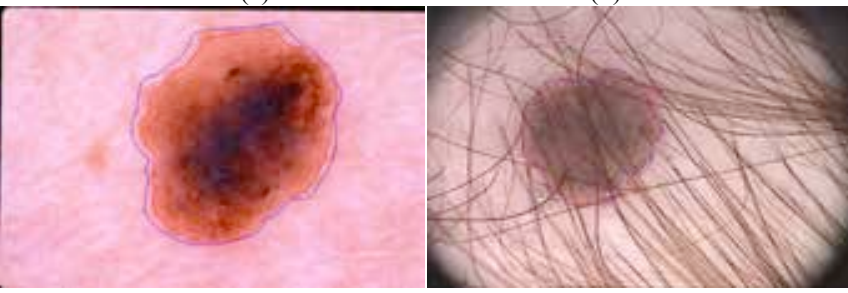

(c)

(d)

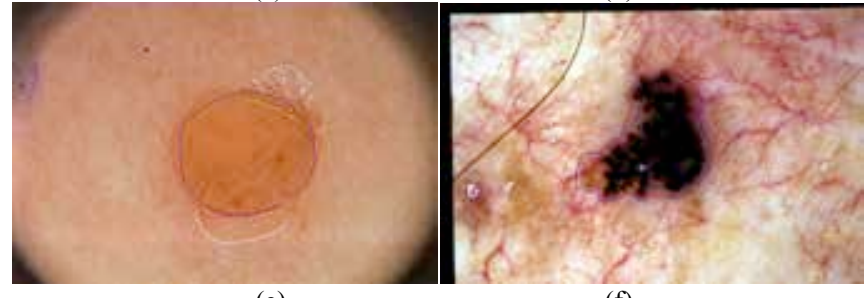

(e)

Fig. 4. Examples of segmentation results: (a)-(c) the segmented boundary (red contour) with the ground truth (blue contour) of the images in Figs. $1 \mathrm{~b}-1 \mathrm{~d}$, with $D\left(I_{0}, I_{1}\right)=0.0787,0.0544,0.1273$, respectively; (d)-(e) the performance of the proposed approach on images with a strong influence of hairs, air bubbles and blood vessels; the skin lesions in (d) and (f) are benign and the skin lesion in (e) are melanoma with $D\left(I_{0}, I_{1}\right)=0.1267,0.1669,0.1668$.

$D\left(I_{0}, I_{1}\right)$ decreases from 0.1641 to 0.0749 . It is also worth pointing out that the parameter $\alpha$ in the proposed algorithm is unlike the common parameters defined in the deformable model-based approaches, which normally have a substantial influence on the segmentation results and require a delicate definition. The parameter $\alpha$ is used because it can adjust the segmentation result flexibly, mimicking manual segmentations of different dermatologists as well as being a user interaction tool in a CAD system.

The proposed algorithm is semi-automatic, as the initial curve needs to be defined manually. The manual initialization was adopted because the performance of the algorithm can be affected when the neighboring region of the initial curve involves parts with significantly dissimilar colors to the normal skin. Meanwhile, external influences, such as black frames and common nevus, often appear simultaneously in the image with the skin lesion; a fully automatic process to exclude these influences is not trivial under the complex background in dermoscopic images and needs considerable pre-processing steps which currently are not robust enough with the existing techniques. Controversially, a manual definition of the initial curve not only helps to exclude these undesired influences, but also makes the segmentation more flexible and robust. Therefore, a manual initialization is a reasonable exchange for full-automation. A common concern is: How is the performance of the algorithm affected by the location and size of the initial curve? There is no restriction on 


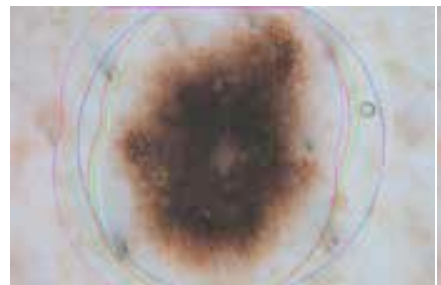

(a)

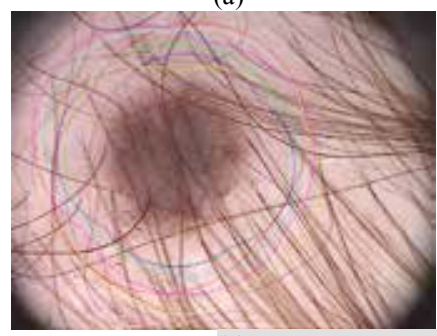

(c)

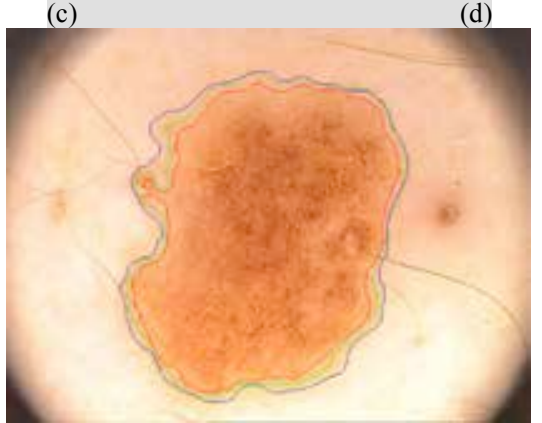

(e)

Fig. 5. (a) Seven initial curves with different shapes and locations in Fig. 1a; (b) The boundaries obtained overlapped with each other, the mean and standard deviation of $D\left(I_{0}, I_{1}\right)$ of these results are 0.0536 and 0.0022 ; (c) Seven initial curves with different shapes and locations in Fig. 4d; (b) The boundaries obtained overlapped with each other; the mean and standard deviation of $D\left(I_{0}, I_{1}\right)$ of these results are 0.1294 and 0.0073 , respectively; (e) Boundaries obtained using $\alpha=0.2$ (green contour) and $\alpha=0.5$ (red contour) with the same initial curve, overlapped with the ground truth (blue contour).

the shape of the initial curve thanks to the deformable model features; but the initial curve must cover the whole region of the skin lesion. An interesting finding is that, with the premise that the neighboring region of the initial curve is not seriously affected by unwanted influence, the initial curve has little influence on the result obtained, except on the convergence time. Fig. 5 illustrates two examples: the different locations of the initial curve caused small changes in the shapes of the final boundary; this is more appreciable in Fig. $4 \mathrm{~d}$ which is a more complex imaging but with the proposed algorithm, the major part of the segmented region remains unchanged both qualitatively and quantitatively. However, if the neighboring region of the initial curve is seriously affected by unwanted influences, the algorithm may not achieve satisfactory results. Meanwhile, the size of the neighboring region of the initial curve determines the statistical information of normal skin around the skin lesions. A larger neighboring region around the initial curve can capture the variations of normal skin more accurately, but is more likely to introduce unwanted influences. A bandwidth of 50 pixels as defined in (12) and (13) were used in the testing of both databases and led to very satisfactory results.

\section{CONCLUSION}

A novel approach based on deformable model is proposed here to segment skin lesions in dermoscopic images. The proposed algorithm combines the information contained in dermoscopic images, and defines the speed function based on the lightness, saturation and color information, with which the evolving curve is guided to stop at the boundary of the skin lesions. Numerical experiments illustrated the effectiveness of the algorithm, and the implementation issues were discussed based on the quantitative analysis.

Compared with other algorithms, the novel approach uses the color information in the dermoscopic images efficiently and carries out the segmentation in a more robust and flexible manner. The implementation of the novel approach is simple and the segmentation results can easily be adjusted or refined through the parameter of color sensitivity. The approach presented here achieves very satisfactory results in challenging image databases and gives sound segmentation for further analysis of skin lesions. The current form of the novel approach is semi-automatic as the initial curves need to be defined manually to avoid negative influence from the complicated imaging background. Future work will continue to enhance the robustness of the approach and study the influence of shape accuracy on the classification of the skin lesions. For example, a pre-processing step such as the pigment separation procedure proposed in [46] and [47] can be a potential way to enhance the performance of a segmentation algorithm. The discussions and techniques referred to in [48] can also be useful when handling dermoscopic images.

\section{ACKNOWLEDGEMENT}

The authors would like to thank Dr. M. Emre Celebi for sharing his image database and comparison data.

\section{REFERENCES}

[1] R. S. Stern, "Prevalence of a history of skin cancer in 2007: results of an incidence-based model," Arch. Dermatol., vol. 146, no. 3, pp. 279-282, 2010.

[2] American Cancer Society. Cancer facts \& figures 2013. Available: http://www.cancer.org/acs/groups/content/@epidemiologysurveilance/d ocuments/document/acspc-036845.pdf.

[3] A. Jemal, R. Siegel, J. Xu, and E. Ward, "Cancer statistics, 2010," CA. Cancer J. Clin., vol. 60, no. 5, pp. 288-296, 2010.

[4] N. Howlader, A. M. Noone, M. Krapcho, J. Garshell, N. Neyman, S. F. Altekruse, C. L. Kosary, M. Yu, J. Ruhl, Z. Tatalovich, H. Cho, A. Mariotto, D. R. Lewis, H. S. Chen, E. J. Feuer, and K. A. Cronin (2012), SEER Cancer Statistics Review, 1975-2010.National Cancer Institute, Bethesa, MD,Available: http://seer.cancer.gov/csr/1975_2009_pops09/.

[5] A. Bleyer, M. O'Leary, R. Barr, and L. A. G. Ries (2006), Cancer Epidemiology in Older Adolescents and Young Adults 15 to 29 Years of Age, Including SEER Incidence and Survival: 1975-2000. National Cancer Institute, Bethesa, MD.

[6] H. Pehamberger, A. Steiner, and K. Wolff. "In vivo epiluminescence microscopy of pigmented skin lesions. I. Pattern analysis of pigmented skin lesions," J. Amer. Acad. Dermatol., vol. 17, no. 4, pp: 571-583, 1987.

[7] C. M. Balch, A. C. Buzaid, S. J. Soong, M. B. Atkins, N. Cascinelli, D. G. Coit, I. D. Fleming, J. E. Gershenwald, A. Jr. Houghton, J. M. Kirkwood, K. M. McMasters, M. F. Mihm, D. L. Morton, D. S. Reintgen, M. I. Ross, A. Sober, J. A. Thompson, and J. F. Thompson, "Final version of the American Joint Committee on Cancer staging system for cutaneous melanoma," J. Clin. Oncol., vol. 19, no. 16, pp: 3635-3648, 2001. 
[8] C. M. Grin, A. W. Kopf, B. Welkovich, R. S. Bart, and M. J. Levenstein. "Accuracy in the clinical diagnosis of malignant melanoma," Arch. Dermatol., vol. 126, no. 6, pp: 763-766, 1990.

[9] M. Binder, M. Schwarz, A. Winkler, A. Steiner, A. Kaider, K. Wolff, and H. Pehamberger, "Epiluminescence microscopy. A useful tool for the diagnosis of pigmented skin lesions for formally trained dermatologists," Arch. Dermatol., vol. 131, no. 3, pp: 286-291, 1995.

[10] H. Kittler, H. Pehamberger, K. Wolff, and M. Binder, "Diagnostic accuracy of dermoscopy,"Lancet Oncol., vol. 3, no. 3, pp: 159-165, 2002.

[11] K. Korotkov and R.Garcia, "Computerized analysis of pigmented skin lesions: a review," Artif. Intell. Med., vol. 56, no. 2, pp: 69-90, 2012.

[12] M. E. Celebi, H. Iyatomi, G. Schaefer, and W. V. Stoecker, "Lesion border detection in dermoscopy images," Comput. Med. Imag. Grap., vol. 33, no. 2, pp: 148-153, 2009.

[13] M. E. Yuksel and M. Borlu, "Accurate segmentation of dermoscopic images by image thresholding based on type-2 fuzzy logic," IEEE T. Fuzzy Syst., vol. 17, no. 4, pp: 976-982, 2009.

[14] M. E. Celebi, H. A. Kingravi, H. Iyatomi, Y. A. Aslandogan, W. V. Stoecker, R. H. Moss, J. M. Malters, J. M. Grichnik, A. A. Marghoob, H. S. Rabinovitz, and S. W. Menzies, "Border detection in dermoscopy images using statistical region merging," Skin Res. Technol., vol. 14, no. 3, pp: 347-353,2008.

[15] H. Iyatomi, H. Oka, M. Saito, A. Miyake, M. Kimoto, J. Yamagami, S. Kobayashi, A. Tanikawa, M. Hagiwara, K. Ogawa, G. Argenziano, H. P. Soyer, and M. Tanaka,"Quantitative assessment of tumor extraction from dermoscopy images and evaluation of computer-based extraction methods for automatic melanoma diagnostic system," Melanoma Res., vol. 16, no. 2, pp: 183-190, 2006.

[16] H. Iyatomi, H. Oka, M. E. Celebi, H. Hashimoto, M. Hagiwara, M. Tanaka, and K. Ogawa, "An improved Internet-based melanoma screening system with dermatologist-like tumor area extraction algorithm," Comput. Med. Imaging Graph., vol. 32, no. 7, pp: 566-579,2008.

[17] Q. Abbas, I. F. Garcia, M. E. Celebi, W. Ahmad, and Q. Mushtaq, "Unified Approach for Lesion Border Detection Based on Mixture Modeling and Local Entropy Thresholding," Skin Res. Technol., vol. 19, no. 3, pp: 314-319, 2013.

[18] M. E. Celebi, Q. Wen, S. Hwang, H. Iyatomi, and G. Schaefer, "Lesion border detection in dermoscopy images using ensembles of thresholding methods," Skin Res. Techno., vol. 19, no. 1, pp: e252-e258, 2013.

[19] P. Schmid, "Segmentation of digitized dermatoscopic images by two-dimensional color clustering," IEEE T. Med. Imaging, vol. 18, no. 2, pp: 164-171, 1999.

[20] G. Schaefer, M. I. Rajab, M. E. Celebi, and H. Iyatomi, "Colour and contrast enhancement for improved skin lesion segmentation," Comput. Med. Imaging Graph., vol. 35, no. 2, pp: 99-104, 2011.

[21] M. E. Celebi, Y. A. Aslandogan, W. V. Stoecker, H. Iyatomi, H. Oka, and X. Chen, "Unsupervised border detection in dermoscopy images," Skin Res. Technol.,vol. 13, no. 4, pp:454-462, 2007.

[22] Q. Abbas, M. E. Celebi, I. Fondon, and M. Rashid,"Lesion border detection in dermoscopy images using dynamic programming," Skin Res. Technol.,vol. 17, no. 1, pp: 91-100, 2011.

[23] Q. Abbas, M. E. Celebi, and I. Fondon, "Skin tumor area extraction using an improved dynamic programming approach," Skin Res. Technol., vol. 18, no. 2, pp: 133-142. 2012.

[24] H. Zhou, G. Schaefer, A. Sadka, and M. E. Celebi, "Anisotropic mean shift based fuzzy c-means segmentation of dermoscopy images," IEEE J. Sel. Top. Signa., vol. 3, no. 1, pp: 26-34, 2009.

[25] A. Wong, J. Scharcanski, and P. Fieguth,"Automatic skin lesion segmentation via iterative stochastic region merging," IEEE T. Inf. Technol. B., vol. 15, no. 6, pp: 929-936, 2011.

[26] J. Gao, J. Zhang, M. G. Fleming, I. Pollak, and A. B. Cognetta, "Segmentation of dermatoscopic images by stabilized inverse diffusion equations," in Proc. IEEE Int. Conf. Image Process, 1998, pp: 823-827.

[27] H. Ganster, A. Pinz, R. Röhrer, E. Wildling, M. Binder, and H. Kittler, "Automated melanoma recognition," IEEE T. Med. Imaging, vol. 20, no. 3 , pp:233-239,2001.

[28] B. Erkol, R. H. Moss, R. J. Stanley, W. V. Stoecker, and E. Hvatum, "Automatic lesion boundary detection in dermoscopy images using gradient vector flow snakes," Skin Res. Technol., vol. 11, no. 1, pp: $17-26,2005$

[29] Q. Abbas, I. Fondón, and M. Rashid,"Unsupervised skin lesions border detection via two-dimensional image analysis," Comput. Methods Programs Biomed., vol. 104, no. 3, pp: e1-15, 2011.
[30] H. Zhou, X. Li, G. Schaefer, M. E. Celebi, and P. Miller, "Mean shift based gradient vector flow for image segmentation," Comput. Vis. Image Und., vol. 117, no. 9, pp. 1004-1016, 2013.

[31] Q. Abbas, M. E. Celebi, and I. F. Garcia, "A novel perceptually-oriented approach for skin tumor segmentation," Int. J. Innov. Comput. I., vol. 8, no. 3, pp. 1837-1848, 2012

[32] M. Mete and N. M. Sirakov, "Lesion detection in dermoscopy images with novel density-based and active contour approaches," $B M C$ Bioinformatics, vol. 11, no. Suppl. 6, pp. S23, 2010.

[33] H. Zhou, G. Schaefer, M. E. Celebi, F. Lin, and T. Liu, "Gradient vector flow with mean shift for skin lesion segmentation," Comput. Med. Imag. Grap., vol. 35, no. 2, pp. 121-127, 2011.

[34] G. A. Hance, S. E. Umbaugh, R. H. Moss, and W. V. Stoeker,"Unsupervised color image segmentation with application to skin tumor borders," IEEE Eng Med Biol.,vol.15, no.1, pp: 104-111, 1996

[35] R. Melli, C. Grana, and R. Cucchiara, "Comparison of color clustering algorithms for segmentation of dermatological images," in Proc. SPIE Med Imaging, 2006, pp: 3S1-3S9.

[36] M. Silveira, J. C. Nascimento, J. S. Marques, A. R. S. Marcal, T. Mendonca, S. Yamauchi, J. Maeda, and J. Rozeira,"Comparison of segmentation methods for melanoma diagnosis in dermoscopy images," IEEE J. Sel. Top. Signa., vol. 3, no. 1, pp: 35-45, 2009.

[37] A. R. A. Ali and T. M. Deserno, "A systematic review of automated melanoma detection in dermatoscopic images and its ground truth data," in Proc. SPIE Med. Imaging, 2012, pp: 8318-8854.

[38] R. C. Gonzalez and R. E. Woods, "Digital Image Processing", $3^{\text {rd }}$ Edition, Prentice Hall. 2007.

[39] M. Sonka, V. Hlavac, and R. Boyle, "Image Processing, Analysis, and Machine Vision", $4^{\text {th }}$ Edition, Cengage Learning. 2014.

[40] S. J. Osher and J. A. Sethian, "Fronts propagating with curvature-dependent speed: algorithms based on Hamilton-Jacobi formulations," J. Comput. Phys., vol. 79, no.1, pp: 12-49, 1998.

[41] Z. Ma, R. N. M. Jorge, andJ. M. R. S. Tavares, "A shape guided C-V model to segment the levator ani muscle in axial magnetic resonance images," Med. Eng. Phys., vol.32, no.7, pp: 766-774, 2010.

[42] N. Otsu, "A threshold selection method from gray-level histograms," IEEE T. Syst. Man. Cyb., vol.9, no.1, pp: 62-66, 1979.

[43] T. Mendonca, P. M. Ferreira, J. S. Marques, A. R. S. Marcal, and J Rozeira. "PH2 - A dermoscopic image database for research and benchmarking," in Proc. IEEE Eng. Med. Biol. Soc., 2013, pp: 5437-5440.

[44] R. Garnavi, M. Aldeen, M. E. Celebi, G. Varigos, and S. Finch, "Border detection in dermoscopy images using hybrid thresholding on optimized color channels," Comput. Med. Imaging Graph., vol. 35, no.2, pp: $105-115,2011$.

[45] H. Zhou, M. Chen, L. Zou, R. Gass, L.Ferris, L.Drogowski, and J. M. Rehg, "Spatially constrained segmentation of dermoscopy images," in Proc. IEEE Int. Symp. Biomed. Imaging, 2008, pp: 800-803.

[46] A. Madooei, M. S. Drew, M. Sadeghi, and M. S. Atkins, "Intrinsic melanin and hemoglobin colour components for skin lesion malignancy detection," Med. Image Comput. Comput. Assist. Interv., vol. 15, pp: 315-322, 2012.

[47] S. Xu, "Skin lesion segmentation by using independent pigment concentration distribution," Journal of image and graphics (in Chinese), vol. 18, no. 11, pp: 1452-1456, 2013.

[48] P. G. Cavalcanti and J. Scharcanski, "Macroscopic pigmented skin lesion segmentation and its influence on lesion classification and diagnosis," Lecture Notes in Computational Vision and Biomechanics, vol. 6, pp: 15-39, 2013.

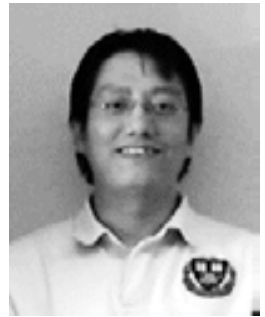

Zhen Ma was born in Shandong, China, in 1982. He received his B.S. and M.S. degrees in applied mathematics from Inner Mongolia University and Beihang University in 2004 and 2007, and a Ph.D. degree in biomedical engineering from the University of Porto, in 2012.

- Currently he is a Researcher at the Instituto de Engenharia Mecânica e Gestão Industrial and the Faculdade de Engenharia da Universidade do Porto, Porto, 
Portugal. His research interests include image processing, biomedical engineering, mathematical modeling, and parallel computing.

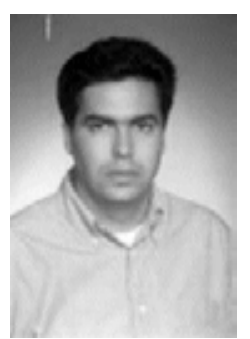

João Manuel R. S. Tavares was born in Porto, Portugal, in 1969. He received a B.S. degree in mechanical engineering in 1992, an M.S. degree in electrical and computer engineering in 1995, and a Ph.D. degree in electrical and computer engineering in 2001, all from the University of Porto.

From 1995 to 2000, he was a Researcher at the Institute of Biomedical Engineering, Porto, Portugal. Since 2001, he has been a Senior Researcher and Project Coordinator at the Laboratory of Optical and Experimental Mechanics of the Institute of Mechanical Engineering and Industrial Management. He was Assistant Professor in the Department of Mechanical Engineering of the Faculty of Engineering of the University of Porto between 2001 and 2011; and since then he has been Associate Professor in the same department. His main research areas include computational vision, computational mechanics, scientific visualization, human-computer interaction and new product development. He is the co-author of more than 550 articles in national and international journals and conferences and co-editor of 30 international books and guest-editor of several special issues of international journals. $\mathrm{He}$ has been co-chairman of various international conferences, for example CompIMAGE 2006/2010/2012/2014, BioDENTAL 2009/ 2012/2014, VipIMAGE 2007/2009/2011/2013/2015 and ICEBS 2013, and of numerous mini-symposia, workshops and thematic sessions. He has been a member of several national and international journal editorial boards and is presently the Editor-in-Chief of the journal "Computer Methods in Biomechanics and Biomedical Engineering: Imaging \& Visualization" and is also Co-editor of the Springer book series "Lecture Notes in Computational Vision and Biomechanics". 